\title{
Simple approximation for the Bethe-ansatz solution of the one-dimensional Fermi gas
}

\author{
M. A. Gusmão* \\ Serin Physics Laboratory, Rutgers University, Piscataway, New Jersey 08854
}

(Received 21 July 1986)

\begin{abstract}
We present a simple approximation scheme for the solution of the integral equations resulting from the Bethe-ansatz diagonalization of the one-dimensional Fermi gas with $\delta$-function attraction. These equations arise for the Hubbard model with attractive interaction in the limit of weak coupling and low density. We obtain the ground-state energy as a function of coupling and density in very good agreement with numerical solutions, as well as a value for the parameter determining the gap in the magnetic excitation spectrum which strongly supports a conjecture of Larkin and Sak.
\end{abstract}

\section{INTRODUCTION}

The one-dimensional Hubbard model with attractive interaction between the electrons is one of the simplest models for a (quasi-) one-dimensional superconductor. In the limit of weak interaction and low electronic density it reduces to a Fermi gas with $\delta$-function attraction. The properties of this and related models have been discussed by a number of authors, ${ }^{1-5}$ using the exact diagonalization of the Hamiltonian by the Bethe ansatz. ${ }^{6}$ The attractive interaction favors formation of electronic pairs, resulting in a ground state with zero magnetization and a gap in the magnetic excitation spectrum. Writing down the Hubbard Hamiltonian for $n$ electrons per site in a linear lattice of $N$ sites as

$H=-T \sum_{i, \sigma}\left(a_{i \sigma}^{\dagger} a_{i+1, \sigma}+a_{i \sigma}^{\dagger} a_{i-1, \sigma}\right)+U \sum_{i} a_{i \uparrow}^{\dagger} a_{i \downarrow}^{\dagger} a_{i \downarrow} a_{i \uparrow}$,

with $T>0$ and $U<0$; the energy gap in the weakcoupling Fermi gas limit $(u=|U| / T \ll<1, \quad n \ll 1$, $u / n \ll 1$ ) was shown ${ }^{1}$ to be given by

$$
\frac{\Delta}{T}=2\left(2 e^{-1}\right)^{1 / 2} u^{2}(n / u)^{3 / 2} e^{\pi^{2} \alpha_{0}} e^{-\pi^{2} n / u},
$$

with a numerical evaluation of the constant $\alpha_{0}$ yielding the value $\alpha_{0} \sim 0.11$.

The same problem was also addressed by Larkin and $\mathrm{Sak}^{7}$ resorting to a renormalization-group calculation. They rederived formula (2) for the gap with an analytical expression for $\alpha_{0}$, namely,

$$
\alpha_{0}=\frac{\ln \pi+1}{2 \pi^{2}}=0.10865 \cdots,
$$

which was obtained by means of an argument of universality and the consequently allowed comparison with the exact solution of the $n=1$ model $^{2}$ Since the latter is not a rigorous approach, it would be interesting to check the result (3) by obtaining a more accurate value of $\alpha_{0}$ from the Bethe-ansatz solution. This was the original aim of this work.

Although we did not obtain $\alpha_{0}$ in closed form, we worked out a scheme of successive approximations to the solution of the relevant integral equation (see below) whose zeroth order already gives $\alpha_{0}$ differing from (3) by about $2 \%$, this difference being reduced to less than $0.4 \%$ in the next step. We believe that this is a good indication of the accuracy of (3). In addition, our method allows us to evaluate the ground-state energy of the Fermi gas for any value of $u / n$. Even the zeroth-order approximation gives this energy as a function of $u / n$ in very good agreement with the numerical results, ${ }^{1}$ as shown in Fig. 1 . We also discuss the possibility of applying this method to the calculation of other quantities, e.g., the energy gap of the Fermi gas as a function of $u / n$, the magnetization in the presence of a magnetic field, and the case of the Hubbard model for arbitrary $u$ and $n$.

\section{BASIC EQUATIONS}

The lowest energy level of the one-dimensional Hubbard model for a fixed value of the total spin component $S_{z}$ is given by the so-called "Lieb and Wu equations.", In the attractive case, defining $s=S_{z} / N$ and setting

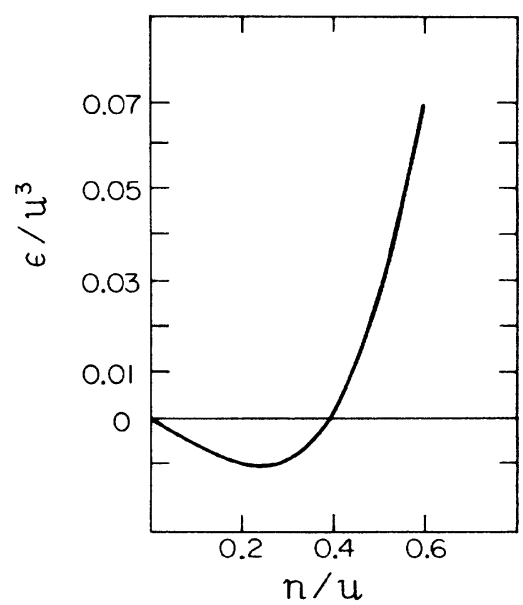

FIG. 1. Ground-state energy of the one-dimensional Fermi gas with attraction. In this scale it is impossible to resolve our zeroth-order approximation from the exact numerical evaluation of Ref. 1. 
$T=1$, these equations can be written ${ }^{5}$ as follows:

$\frac{E}{N}=-\left[\frac{n}{2}-s\right] u-2 \int_{-Q}^{Q} \rho(k) \cos k d k$,

$2 \pi \rho(k)=1+\cos k \int_{-B}^{B} \frac{8 u \sigma(\lambda)}{u^{2}+16(\sin k-\lambda)^{2}} d \lambda$,

$2 \pi \sigma(\lambda)+\int_{-B}^{B} \frac{4 u \sigma\left(\lambda^{\prime}\right)}{u^{2}+4\left(\lambda-\lambda^{\prime}\right)^{2}} d \lambda^{\prime}$

$$
=\int_{-Q}^{Q} \frac{8 u \rho(k)}{u^{2}+16(\sin k-\lambda)^{2}} d k,
$$

$\int_{-Q}^{Q} \rho(k) d k=1-2 s$,

$\int_{-B}^{B} \sigma(\lambda) d \lambda=\frac{n}{2}-s$.

For the ground state $(s=0)$ the right-hand side of (7) becomes unity, yielding $Q=\pi$ and allowing for the elimination of $\rho(k)$ from the set $(4)-(8)$, which reduces to ${ }^{1}$

$$
\begin{aligned}
& \frac{E}{N}=-\frac{n}{2} u-2 \int_{-B}^{B} \sigma(\lambda) g_{2}(\lambda) d \lambda, \\
& 2 \pi \sigma(\lambda)+\int_{-B}^{B} \frac{4 u \sigma\left(\lambda^{\prime}\right)}{u^{2}+4\left(\lambda-\lambda^{\prime}\right)^{2}} d \lambda^{\prime}=g_{0}(\lambda), \\
& \int_{-B}^{B} \sigma(\lambda) d \lambda=\frac{n}{2}
\end{aligned}
$$

where

$$
g_{n}(\lambda)=\frac{1}{2 \pi} \int_{-\pi}^{\pi} \frac{8 u \cos ^{n} k}{u^{2}+16(\sin k-\lambda)^{2}} d k .
$$

Finally, in the Fermi gas limit $u \rightarrow 0, n \rightarrow 0$, with $u / n$ constant, Eqs. (9) and (10) become ${ }^{1}$

$$
\begin{aligned}
& \epsilon \equiv \frac{E}{N}+2 n=-\frac{n u^{2}}{16}+2 \int_{-B}^{B} \lambda^{2} \sigma(\lambda) d \lambda, \\
& 2 \pi \sigma(\lambda)+\int_{-B}^{B} \frac{4 u \sigma\left(\lambda^{\prime}\right)}{u^{2}+4\left(\lambda-\lambda^{\prime}\right)^{2}} d \lambda^{\prime}=2 .
\end{aligned}
$$

Introducing the notation

$\kappa \equiv \frac{u}{2 B}, x \equiv \frac{\lambda}{B}, f(x ; \kappa) \equiv \pi \sigma(\lambda), \quad K(x ; \kappa) \equiv \frac{\kappa / \pi}{\kappa^{2}+x^{2}}$,

we can rewrite Eqs. (14) and (11) in the form

$$
\begin{aligned}
& f(x ; \kappa)+\int_{-1}^{1} d x^{\prime} K\left(x-x^{\prime} ; \kappa\right) f\left(x^{\prime} ; \kappa\right)=1, \\
& \int_{-1}^{1} d x f(x ; \kappa)=\frac{\pi}{2}(n / B) .
\end{aligned}
$$

Equation (16) also appears in the problem of calculating the capacity of a circular parallel plate condenser, ${ }^{8}$ where it is known as Love's integral equation. Numerical solutions for various values of $\kappa$ were first reported by Nomu$\mathrm{ra}^{9}$ and later corrected by Cooke. ${ }^{10}$ The Nomura-Cooke values are shown in Table I.

Given $f(x ; \kappa)$ as a solution of Eq. (16), the dependence of the parameter $B$ on $u$ and $n$ is determined by (17). In the weak-coupling limit $u / n \ll 1(\kappa<1)$, Krivnov and Ovchinnikov ${ }^{1}$ have found
TABLE I. Comparison between our zeroth- and first-order approximations for the integral $I(\kappa)$, Eq. (24), and the exact (numerical) results reported in Ref. 8.

\begin{tabular}{lccc}
\hline$\kappa$ & $I_{0}(\kappa)$ & $I_{1}(\kappa)$ & $I(\kappa)$ (exact) \\
\hline 0 & $\frac{1}{2}$ & $\frac{1}{2}$ & $\frac{1}{2}$ \\
0.4 & 0.6019 & 0.6023 & 0.6027 \\
0.6 & 0.6359 & 0.6363 & 0.6364 \\
0.8 & 0.6652 & 0.6655 & 0.6656 \\
1.0 & 0.6910 & 0.6911 & 0.6912 \\
1.2 & 0.7137 & 0.7138 & 0.7138 \\
1.5 & 0.7429 & 0.7430 & 0.7430 \\
2.0 & 0.7817 & 0.7817 & 0.7817 \\
2.5 & 0.8113 & 0.8113 & 0.8113 \\
3.0 & 0.8342 & 0.8342 & 0.8342 \\
5.0 & 0.8896 & 0.8896 & 0.8896 \\
10.0 & 0.9405 & 0.9405 & 0.9405 \\
\hline \hline
\end{tabular}

$$
\frac{B}{u}=\frac{\pi}{2}\left(\frac{n}{u}\right)-\frac{1}{4 \pi} \ln \left(\pi \frac{n}{u}\right)-\frac{\pi}{2} \alpha_{0}+O\left(\frac{\ln (n / u)}{n / u}\right) \text {, }
$$

where the constant $\alpha_{0}$ is the same as that which appears in Eq. (2) for the energy gap. Our aim is thus to solve Eq. (16) for $f(x ; \kappa<1)$ and integrate this solution in (17) to check Eq. (18) and determine the value of $\alpha_{0}$.

\section{ZEROTH-ORDER APPROXIMATION}

We can easily solve Eq. (16) in the two extreme limits $\kappa=0$ and $\kappa \rightarrow \infty$. In the latter the kernel goes to zero, while in the former it becomes a $\delta$ function. This yields

$$
\begin{aligned}
& f(x ; 0)= \begin{cases}\frac{1}{2}, & x^{2}<1 \\
1, & x^{2}>1\end{cases} \\
& f(x ; \infty)=1 .
\end{aligned}
$$

For $\kappa$ small, $K\left(x-x^{\prime} ; \kappa\right)$ is sharply peaked around $x=x^{\prime}$ while $f(x ; \kappa)$ is a smooth function of $x$ except for the two small regions around $x= \pm 1$. For this reason, we can try the following approximation:

$\int_{-1}^{1} d x^{\prime} K\left(x-x^{\prime} ; \kappa\right) f\left(x^{\prime} ; \kappa\right) \simeq f(x ; \kappa) \int_{-1}^{1} d x^{\prime} K\left(x-x^{\prime} ; \kappa\right)$.

Substituting (21) into (16) we get the approximate solution $^{11}$

$$
f_{0}(x ; \kappa)=\frac{1}{1+t(x ; \kappa)},
$$

with

$$
\begin{aligned}
t(x ; \kappa) & \equiv \int_{-1}^{1} d x^{\prime} K\left(x-x^{\prime} ; \kappa\right) \\
& =\frac{1}{\pi} \tan ^{-1}\left[\frac{1+x}{\kappa}\right)+\frac{1}{\pi} \tan ^{-1}\left(\frac{1-x}{\kappa}\right) .
\end{aligned}
$$

We can check that the limits (19) and (20) are satisfied. Indeed, it happens that this approximation is much better for large $\kappa$, since in this limit, although the peak in 
$K\left(x-x^{\prime} ; \kappa\right)$ becomes smeared, $f(x ; \kappa)$ becomes much smoother, without the rapid variations around the points $x= \pm 1$. The limit $\kappa=0$ is also correct, for the variation of $f(x ; \kappa)$ is finite while the range in which this variation occurs goes to zero; thus its contribution to the integral in (21) vanishes.

In order to obtain the parameter $B$ in Eq. (17) we need to evaluate

$$
I(\kappa) \equiv \frac{1}{2} \int_{-1}^{1} d x f(x ; \kappa)=\int_{0}^{1} d x f(x ; \kappa)
$$

In our zeroth-order approximation we have

$$
I_{0}(\kappa)=\int_{0}^{1} \frac{d x}{1+t(x ; \kappa)},
$$

with $t(x ; \kappa)$ given by Eq. (23). Some values of this integral (numerically evaluated) are listed in Table I. Evidently the agreement with the exact results is remarkable for such a crude approximation. It also presents the correct asymptotic behavior for large $\kappa$ and the exact limiting value $I_{0}(\kappa=0)=\frac{1}{2}$. The behavior of $I_{0}(\kappa)$ for small $\kappa$, as shown in the Appendix, is described by the relation

$$
I_{0}(\kappa)=\frac{1}{2}-\frac{\kappa \ln \kappa}{4 \pi}+\frac{\pi}{2} \alpha_{0}^{(0)} \kappa+O\left(\kappa^{2} \ln \kappa\right),
$$

with

$$
\begin{aligned}
\alpha_{0}^{(0)} & =\frac{4}{\pi} \int_{0}^{\infty} d x\left(\frac{1}{3+(2 / \pi) \tan ^{-1} x}-\frac{1}{8 \pi(x+2)}-\frac{1}{4}\right) \\
& =0.10644 \cdots .
\end{aligned}
$$

Equations (26), (24), and (17) reproduce the form (18) for $B / u$, with the approximate value of $\alpha_{0}$ given by (27). Comparison with the expected exact value (3) shows a difference of about $2 \%$.

Within the same approximation we also evaluated the ground-state energy (13) for arbitrary $u / n$. The results are plotted in Fig. 1 together with the values obtained by a numerical solution ${ }^{1}$ of Eq. (14) (in which $\lambda$ was turned into a discrete variable, thereby transforming the integral equation into a set of algebraic equations). The comparison shows again an agreement that is remarkable, given the simplicity of the approximation.

\section{SUCCESSIVE APPROXIMATION SCHEME}

Since $f_{0}(x ; \kappa)$, as defined by Eq. (22), is a reasonable approximate solution of (16), it can be used as the starting point for either an iterative solution or a successive approximation scheme in which the same approximation is recursively employed for the corrections to $f_{0}(x ; \kappa)$. We chose this latter approach because it showed faster convergence when compared to the iterative solution, which is known ${ }^{8}$ to be a convergent scheme for this kind of integral equation.

Writing $f(x ; \kappa)=f_{0}(x ; \kappa)+\Delta f_{0}(x ; \kappa)$ in Eq. (16), the correction term must satisfy the equation

$\Delta f_{0}(x ; \kappa)+\int_{-1}^{1} d x^{\prime} K\left(x-x^{\prime} ; \kappa\right) \Delta f_{0}\left(x^{\prime} ; \kappa\right)=h_{0}(x ; \kappa)$,

where $h_{0}(x ; \kappa)=1-f_{0}(x ; \kappa)-\int_{-1}^{1} d x^{\prime} K\left(x-x^{\prime} ; \kappa\right) f_{0}\left(x^{\prime} ; \kappa\right)$

is the "error" function, which measures the failure of $f_{0}(x ; \kappa)$ to fulfill $(16)$.

We again expect $\Delta f_{0}(x ; \kappa)$ to be smooth except for the narrow regions around $x= \pm 1$. Then, using approximation (21) for $\Delta f_{0}(x ; \kappa)$ in Eq. $(28)$ and calling $f_{1}(x ; \kappa)$ the resulting solution, we have

$$
f_{1}(x ; \kappa)=\frac{h_{0}(x ; \kappa)}{1+t(x ; \kappa)}=f_{0}(x ; \kappa) h_{0}(x ; \kappa) .
$$

The process can now be continued by writing $\Delta f_{0}(x ; \kappa)=f_{1}(x ; \kappa)+\Delta f_{1}(x ; \kappa)$ and applying the same treatment to the integral equation determining $\Delta f_{1}(x ; \kappa)$, and so on. We then obtain

$$
\begin{aligned}
& f(x ; \kappa)=f_{0}(x ; \kappa)+f_{1}(x ; \kappa)+f_{2}(x ; \kappa)+\cdots, \\
& f_{n+1}(x ; \kappa)=f_{0}(x ; \kappa) h_{n}(x ; \kappa), \\
& \begin{array}{rl}
h_{n+1}(x ; \kappa)= & h_{n}(x ; \kappa)-f_{n+1}(x ; \kappa) \\
& \quad-\int_{-1}^{1} d x^{\prime} K\left(x-x^{\prime} ; \kappa\right) f_{n+1}\left(x^{\prime} ; \kappa\right), \\
n & n=0,1,2,3, \ldots,
\end{array}
\end{aligned}
$$

with $f_{0}(x ; \kappa)$ and $h_{0}(x ; \kappa)$ given by Eqs. (22) and (29), respectively. For the integral $I(\kappa)$, Eq. (24), we now have

$$
\begin{gathered}
I(\kappa)=I_{0}(\kappa)+\sum_{n=1}^{\infty} \Delta I_{n}(\kappa), \\
\Delta I_{n}(\kappa)=\int_{0}^{1} d x f_{n}(x ; \kappa) .
\end{gathered}
$$

Some values of $I_{1}(\kappa)=I_{0}(\kappa)+\Delta I_{1}(\kappa)$ are listed in Table I, showing an improved agreement with the exact values, even for $\kappa$ quite small.

From the comments following Eq. (27) we see that both the constant and the logarithmic terms in $I(\kappa)$ are already correctly given by $I_{0}(\kappa)$. So, in the limit of small $\kappa$ the corrections $\Delta I_{n}(\kappa)$ directly yield corrections to $\alpha_{0}$. We can write the $n$th approximation for this parameter as

$$
\alpha_{0}^{(n)}=\alpha_{0}^{(0)}+\sum_{m=1}^{n} \Delta \alpha_{0}^{(m)},
$$

where

$$
\Delta \alpha_{0}^{(m)}=\lim _{\kappa \rightarrow 0} \frac{2 \Delta I_{m}(\kappa)}{\pi \kappa} .
$$

In the first approximation we obtain the value $\alpha_{0}^{(1)}=0.10825$, which differs by less than $0.4 \%$ from the renormalization-group ${ }^{7}$ result (3). So, although our scheme does not yield an analytical verification of the latter, there are good indications that it can be approached to the desired precision.

\section{CONCLUSIONS AND POSSIBLE EXTENSIONS}

In summary, we have developed a method of successive approximations to the solution of the Fredholm integral equation of the second kind, ${ }^{8}$ Eq. (14) [or (16)], that appears in the Bethe-ansatz solution of the one-dimensional Fermi gas with attractive interaction. With this simple 
scheme we were able to reproduce some of the results obtained by Krivnov and Ovchinnikov ${ }^{1}$ who used much more involved mathematical methods. In particular, we obtained, even in the simplest approximation, a very accurate plot of the Fermi gas ground-state energy as a function of $u / n$ (Fig. 1). Furthermore, our results strongly indicate the correctness of the renormalization-group ${ }^{7}$ result (3) for the parameter $\alpha_{0}$ appearing in the expression for the energy gap in the weak-coupling limit, Eq. (2). A plot of this equation is presented in Fig. 2 .

We can also speculate on the possibility of using the same method to study other properties of the Fermi gas, or even the Hubbard model for more general parameters, since all the integrals appearing in the Bethe-ansatz solution present essentially the same form. Indeed, we tried to obtain the $u / n$ dependence of the energy gap $\Delta$ for the Fermi gas, using the equations of Krivnov and Ovchinnikov: ${ }^{1}$

$$
\begin{aligned}
& \Delta=\frac{1}{8} u^{2}-2 B^{2}+4 \int_{-B}^{B} \lambda \psi(\lambda) d \lambda \\
& 2 \pi \psi(\lambda)+\int_{-B}^{B} \frac{4 u \psi\left(\lambda^{\prime}\right)}{u^{2}+4\left(\lambda-\lambda^{\prime}\right)^{2}} d \lambda^{\prime} \\
& =\pi+4 \tan ^{-1}\left(\frac{4 \lambda}{u}\right)-2 \tan ^{-1}\left(\frac{2(\lambda-B)}{u}\right)
\end{aligned}
$$

The zeroth-order approximation, as shown in Fig. 2, is only good for small $n / u$ (large $\kappa$ ), departing from the exact (numerical) result as $\kappa$ becomes small. This can be explained by the fact that the right-hand side of (36), in contrast with (14), presents fast variations for $\lambda \sim 0$ or $\lambda \sim B$ when $u$ is small. As a result, $\psi(\lambda)$ is no longer a very smooth function of $\lambda$, violating the basic assumption of the approximation (21). The same difficulty is expected to arise in the Hubbard model for general parameters [Eq. (10)], or in the study of the magnetization in the presence of a magnetic field, ${ }^{4,5}$ when it is necessary to treat the case of $s \neq 0$ in Eqs. (4)-(8). We still expect the higherorder approximations to become increasingly accurate.

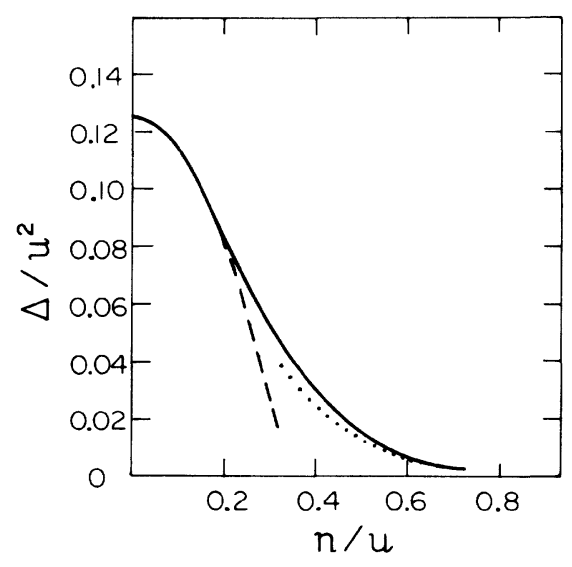

FIG. 2. Energy gap of the one-dimensional Fermi gas with attraction. The solid curve is the numerical result (Ref. 1), the dashed curve is our zeroth-order approximation for Eqs. (35) and (36), and the dotted curve is the result of Eq. (2) with $\alpha_{0}$ given by (3).
However, since the final results involve numerical evaluation of integrals in which the number of variables increases with the order of approximation, it appears that the method will, at least, lose its simplicity.

\section{ACKNOWLEDGMENTS}

The author is greatly indebted to Joseph Sak for the suggestion of the problem as well as for fruitful discussions, and to Timothy Ziman for a critical reading of the manuscript. This work was supported in part by Conselho Nacional de Desenvolvimento Científico e Technológico, (CNPq) Brazil, and by the U.S. National Science Foundation (NSF) under Grant No. DMR-8216223.

\section{APPENDIX}

We shall derive Eqs. (26) and (27) as the small- $\kappa$ limit of Eq. (25). In this limit we can make the approximation

$$
\frac{1}{\pi} \tan ^{-1}\left(\frac{1+x}{\kappa}\right) \simeq \frac{1}{2}-\frac{\kappa}{\pi(1+x)}
$$

in the first term of $t(x ; \kappa)$, Eq. (23), since we are integrating over positive values of $x$. Keeping only terms up to first order in $\kappa$, except for the "dangerous" second term in (23), we have

$$
\begin{aligned}
& \frac{1}{1+t(x ; \kappa)} \simeq \frac{2}{3+\frac{2}{\pi} \tan ^{-1}\left(\frac{1-x}{\kappa}\right)}+\frac{\kappa}{4 \pi(1+x)}, \\
& I_{0}(\kappa) \simeq 2 \int_{0}^{1} d x \frac{1}{3+\frac{2}{\pi} \tan ^{-1}\left(\frac{x}{\kappa}\right)}+\frac{\kappa}{4 \pi} \ln 2 .
\end{aligned}
$$

A change of variable in the last integral yields

$$
I_{0}(\kappa) \simeq 2 \kappa \int_{0}^{1 / \kappa} d x \frac{1}{3+(2 / \pi) \tan ^{-1} x}+\frac{\kappa}{4 \pi} \ln 2
$$

For large $x$ we see that

$$
\frac{1}{3+(2 / \pi) \tan ^{-1} x} \rightarrow \frac{1}{4}+\frac{1}{8 \pi x} .
$$

Subtracting this limiting value and introducing a small- $x$ cutoff (since the integrand is finite for $x=0$ ),

$$
\begin{aligned}
I_{0}(\kappa) \simeq & \frac{1}{2}-\frac{\kappa \ln \kappa}{4 \pi}+\frac{\kappa \ln (2 / a)}{4 \pi} \\
+2 \kappa \int_{0}^{1 / \kappa} d x & {\left[\frac{1}{3+(2 / \pi) \tan ^{-1} x}\right.} \\
& \left.-\frac{1}{8 \pi(x+a)}-\frac{1}{4}\right]
\end{aligned}
$$

Finally, choosing $a=2$ and evaluating the last integral at $\kappa=0$, we arrive at Eqs. (26) and (27). 
*Permanent address: Instituto de Física, Universidade Federal do Rio Grande do Sul, 90049 Porto Alegre, Rio Grande do Sul, Brazil.

${ }^{1}$ V. Ya. Krivnov and A. A. Ovchinnikov, Zh. Eksp. Teor. Fiz. 67, 1568 (1974) [Sov. Phys.-JETP 40, 781 (1974)].

${ }^{2}$ E. H. Lieb and F. Y. Wu, Phys. Rev. Lett. 20, 1445 (1968).

${ }^{3}$ C. N. Yang, Phys. Rev. Lett. 19, 1312 (1967).

${ }^{4}$ M. Takahashi, Prog. Theor. Phys. 42, 1098 (1969).

${ }^{5}$ T. B. Bahder and F. Woynarovich, Phys. Rev. B 33, 2114 (1986).
${ }^{6}$ H. A. Bethe, Z. Phys. 71, 205 (1931).

${ }^{7}$ A. I. Larkin and J. Sak, Phys. Rev. Lett. 39, 1025 (1977).

${ }^{8}$ I. N. Sneddon, Mixed Boundary Value Problems in Potential Theory (North-Holland, Amsterdam, 1966).

${ }^{9}$ Y. Nomura, Proc. Phys. Math. Soc. Jpn. 23, 168 (1941).

${ }^{10}$ J. C. Cooke, Z. Angew. Math. Mech. 38, 349 (1958).

${ }^{11} \mathrm{We}$ have been told by Thomas Bahder (private communication) that Paul Blatz independently derived a similar approximation in an unpublished work. 